5. Wilton, S. J. E., "SMAP: Heterogeneous Technology Mapping for Area Reduction in FPGAs with Embedded Memory Arrays", in Proceedings of the ACM/SIGDA International Symposium on Field-Programmable Gate Arrays, Feb. 1998.

6. Cong, J. and Xu, S., "Technology Mapping for FPGAs with Embedded Memory Blocks", in Proceedings of the ACM/SIGDA International Symposium on FieldProgrammable Gate Arrays, Feb. 1998.

7. Heile, F. and Leaver, A., "Hybrid Product Term and LUT Based Architectures Using Embedded Memory Blocks", in Proceedings of the ACM/SIGDA International Symposium on Field-Programmable Gate Arrays, pp. 13-16, Feb. 1999.

8. Anderson, J. H. and Brown, S. D., "Technology Mapping for Large Complex PLDs", in Proceedings of the 35th ACM/IEEE Design Automation Conference, pp. 698-703, 1998.

9. Cong, J., Huang, H., and Yuan, X., "Technology Mapping for k/m-Macrocell Based FPGAs", in Proceedings of the ACM/SIGDA International Symposium on FireldProgrammable Gate Arrays, pp 51-59, Feb. 2000.

10. Krishnamoorthy, S., Swaminathan, S., and Tessier, R., "Area-Optimized Technology Mapping for Hybrid FPGAs," in Proceedings of the International Conference on Field Programmable Logic and Applications, August 2000.

11. Heile, F., "Programmable Logic Array Device with Random Access Memory Configurable as Product Terms," U.S. Patent No. 6020759, Feb. 2000. 


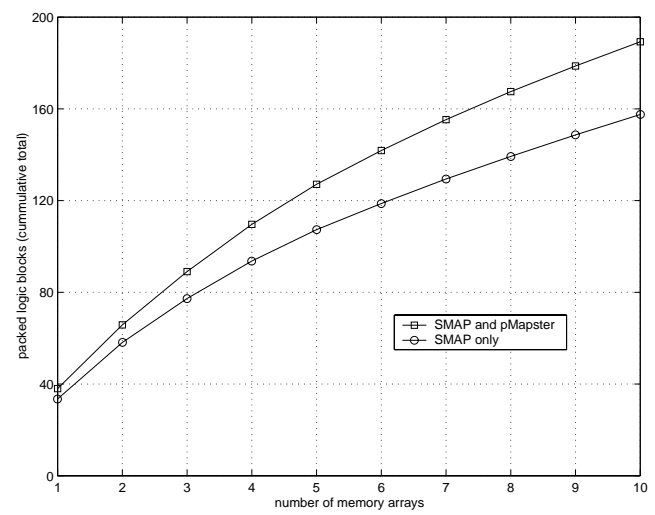

Fig.10. A performance comparison between SMAP and SMAP with pMapster

sharing did not substantially increase the amount of logic that could be packed into each memory array. In fact, compared with no sharing and over 10 memory arrays, the amount of packed logic increased by only $1 \%$ with eight parallel expanders. We also examined how macrocell granularity affected packed logic and found that increasing granularity also decreased the amount of logic that was packed into the memory array because of decreased flexibility in the number of macrocell outputs. Lastly, we examined how pMapster could enhance SMAP's performance since SMAP does not perform as well at higher numbers of memory arrays. We found that pMapster (working with SMAP) increased the amount of packed logic by between $13.4 \%$ and $39.8 \%$ for each memory array and by $20.1 \%$ over 10 arrays.

\section{Acknowledgments}

This work was supported by the British Columbia Advanced Systems Institute and the Natural Sciences and Engineering Research Council of Canada.

\section{References}

1. Xilinx, Inc., XC4000E and XC4000X Field Programmable Gate Arrays Datasheet, ver. 1.6, May 1999.

2. Altera Corp., APEX20K Programmable Logic Device Family Datasheet, ver. 3.3, Jan. 2001.

3. Xilinx, Inc., Virtex-E 1.8V Field-Programmable Gate Arrays Datasheet, ver. 1.3, Feb. 2000.

4. Ngai, T., Rose, J., and Wilton, S. J. E., "An SRAM Programmable FieldConfigurable Memory," in Proceedings of the IEEE 1995 Custom Integrated Circuits Conference, pp.499-502, May 1995. 
that the combination of the two algorithms works better than SMAP alone, especially as the number of arrays increases.

We estimate that adding product term capability to an embedded memory array increases its area by approximately $18.4 \%$. However, adding product term capability also increases the amount of logic that can be packed in the array by between $13.4 \%$ to $39.8 \%$. Thus having product term capability is a worthwhile feature.

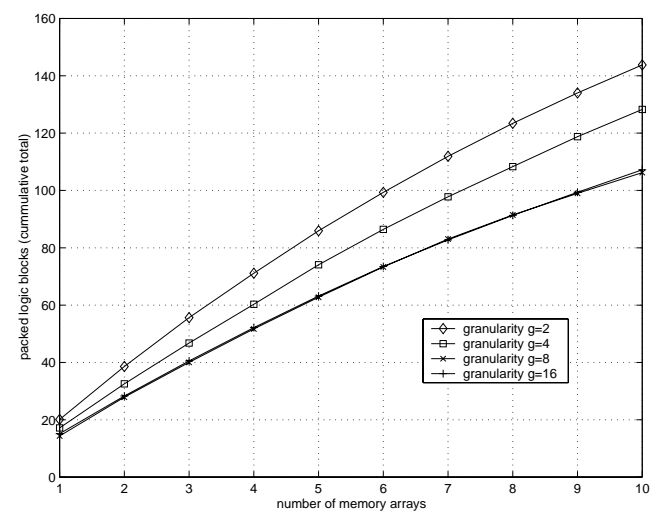

Fig. 8. The effect of granularity on the number of packed logic blocks

\begin{tabular}{|c||c|c|c|c|c|c|c|c|c|c|}
\hline \multicolumn{1}{|c||}{} & \multicolumn{10}{c|}{ Number of Memory Arrays } \\
\cline { 2 - 11 } & 1 & 2 & 3 & 4 & 5 & 6 & 7 & 8 & 9 & 10 \\
\hline \hline no sharing & 20.4 & 38.8 & 56.0 & 71.5 & 86.3 & 99.6 & 111.9 & 123.1 & 133.3 & 142.6 \\
\hline sharing, $p=1$ & 20.5 & 39.1 & 55.9 & 71.6 & 86.6 & 100.5 & 112.5 & 123.8 & 133.8 & 143.3 \\
\hline sharing, $p=2$ & 20.5 & 39.1 & 56.0 & 71.7 & 86.8 & 100.8 & 113.0 & 124.1 & 134.4 & 143.9 \\
\hline sharing, $p=4$ & 20.6 & 39.2 & 56.0 & 71.7 & 86.8 & 100.8 & 113.0 & 124.2 & 134.5 & 144.0 \\
\hline sharing, $p=8$ & 20.6 & 39.2 & 56.1 & 71.8 & 86.9 & 100.9 & 113.1 & 124.3 & 134.6 & 144.0 \\
\hline
\end{tabular}

Fig. 9. The effect of sharing on the number of packed logic blocks (numbers are geometric means over all benchmarks)

\section{Conclusions}

In this paper we have proposed a new architecture for facilitating the sharing of product terms between adjacent macrocells. In order to evaluate this new architecture, a new technology mapping tool, pMapster, was created to take advantage of this enhancement. Experimental results show that product term 


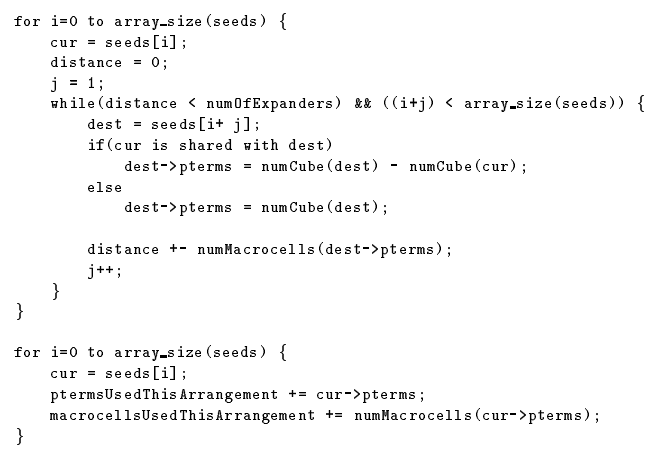

Fig. 7. Pseudocode listing for finding the fitness of a particular arrangement of seeds

mark suite. Initially, all circuits were optimized using SIS and technology mapped into 4-LUTs using Flow Map.

We first examined the effect of macrocell granularity, as defined in section 2.2. We fixed the total number of product terms (columns of the memory) at 32 , but varied how many of the product terms are associated with each macrocell. The number of inputs (literals) to each memory was held at 32. Clearly, the more product terms per macrocell, the wider the function that can be implemented by each macrocell. On the other hand, more product term per macrocell means there are fewer macrocells (and hence outputs) in the memory. Figure 8 shows the number of logic blocks that can be packed into memory arrays, as a function of the number of arrays, for four different granularities. As can be seen, the amount of logic that can be packed into the arrays decreases as the granularity (number of product terms per macrocell) increases. The best choice is to have two product terms per macrocell.

Second, we considered the ability of the parallel expanders (sharing architecture) to increase the amount of logic that can be implemented by each memory. We assumed an architecture in which each memory has 32 inputs (literals) and 16 macrocells of two product terms each. The number of parallel expanders was varied from 1 to 8 . For comparison, we also included the non-sharing base architecture. As shown in Figure 9, as we increase the number of parallel expanders, the amount of logic we can pack into each memory increases slightly.

Finally, we compared the overall packing density for two cases: (1) using SMAP alone (which implements logic in the memory arrays by configuring them as ROMs) and (2) using a combination of SMAP and the pMapster algorithm. In the second case, we use both SMAP and pMapster on each memory array, and choose the better of the two results (recall that each memory array can be configured to operate as a ROM or as a collection of pterms). Figure 10 shows 


\subsection{Extending the Algorithm to Handle Sharing}

The algorithm in Figure 5 assigns output signals to the output macrocells in the memory arbitrarily. When using the product term sharing architectures outlined in section 2, however, the manner in which the outputs are assigned to macrocells can affect the number of macrocells required. This is shown in Figure 6.

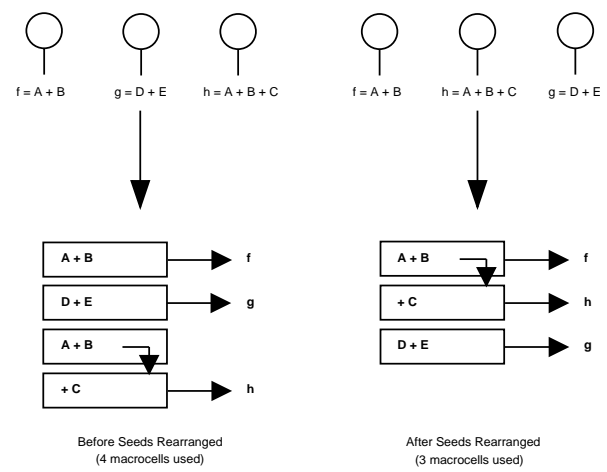

Fig. 6. How rearranging seeds leads to better use of macrocells when sharing is possible

In order to take this into account, we first assign the outputs to macrocells arbitrarily, as in Figure 5. We then use a greedy swapping algorithm to improve the assignment. In order to evaluate the fitness of a given assignment, we step through each output in order and determine if an output can be shared with any of its neighbouring outputs. In the example shown in Figure 6, we can see that output $(A+B)$ shares product terms with output $(A+B+C)$. Thus, if placed adjacent to each other, they could share their common product terms.

We also need to keep track of how many neighbouring outputs we consider, since clearly if an output requires more than one macrocell to implement it, that "steals" one or more of the available parallel expanders. Therefore, a "macrocell distance" is calculated which determines how many outputs are to be considered. Figure 7 shows the pseudocode of the fitness function.

Using the above fitness function, the algorithm rearranges the output assignment by randomly swapping two outputs. If the new assignment results in a lower overall cost, the move is accepted, otherwise, it is not. This is repeated $n^{2}$ times, where $n$ is the number of outputs being considered. We experimented with varying values of repetitions, and found that increasing the number of repetitions to $n^{3}$ did not improve the results.

\section{Experimental Results and Discussion}

To evaluate the proposed macrocell architectures, we used 17 large benchmark circuits from the Microelectronics Corporation of North Carolina (MCNC) bench- 
collapse fanin nodes into the seed. For nodes with multiple fanins, we choose the fanin as to minimize the number of inputs and product terms of the resultant collapsed node.

If the fanin has only one output (the seed node), we can remove the fanin as we collapse it. If the fanin has more than one fanout (ie. feeds a node other than the seed node), then we can still remove the fanin node, as long as we use an output of the memory array to implement the fanin signal. In this way, we can collapse beyond the maximum fanout-free cone rooted at the seed node.

Fanin nodes are collapsed into the seed until the number of product terms, inputs, or outputs in the memory is exceeded. We then repeat the algorithm for each seed node, and choose the seed node that results in the maximum number of nodes being deleted.

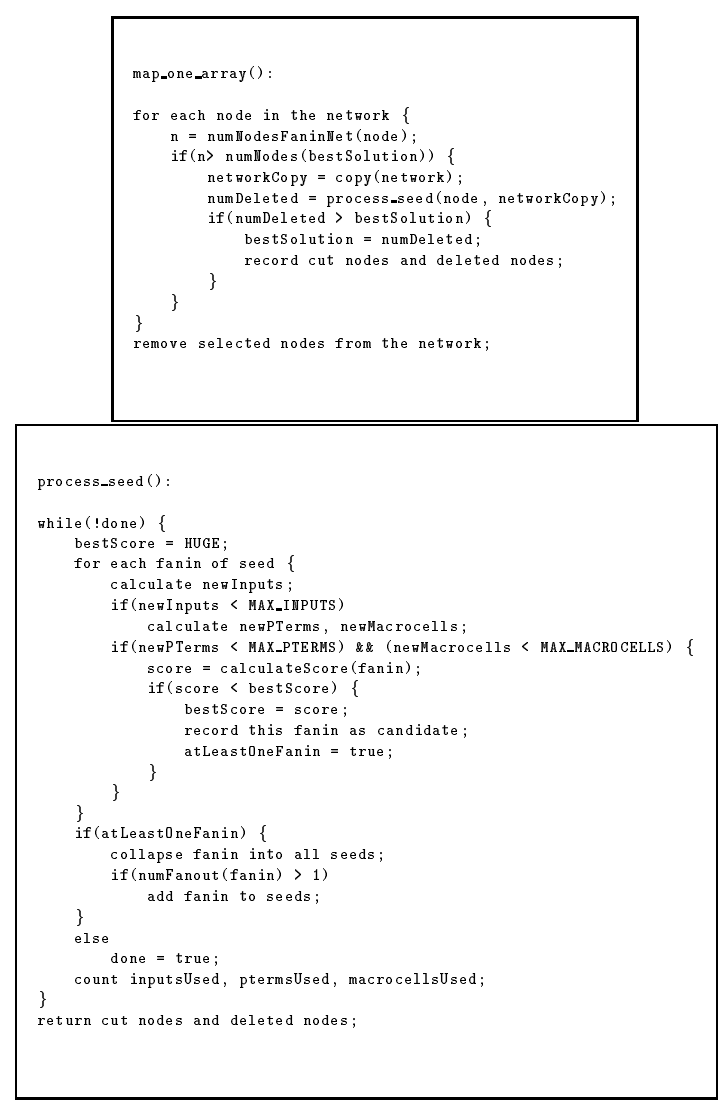

Fig. 5. Pseudocode listing for pMapster Algorithm 
be used by at most two macrocells (the generating macrocell and the macrocell adjacent to it, assuming macrocell output sharing exists). In certain cases, this could lead to an inefficient use of macrocells since certain product terms may be repeated. Increasing the number of parallel expanders could increase product term usage efficiency, since product term repetition can be reduced.

In cases where the product term block must implement a number of functions which are similar, although not necessarily incrementally similar as in the previous example, having a macrocell capable of sharing with the next 2, 4, 6, or more macrocells may be useful. A macrocell can then be used to generate the common product terms (in a set of functions) and share them with several other macrocells. Figure 4a) shows how this could be useful. Figure 4b) shows a basic macrocell with enhanced parallel expanders.
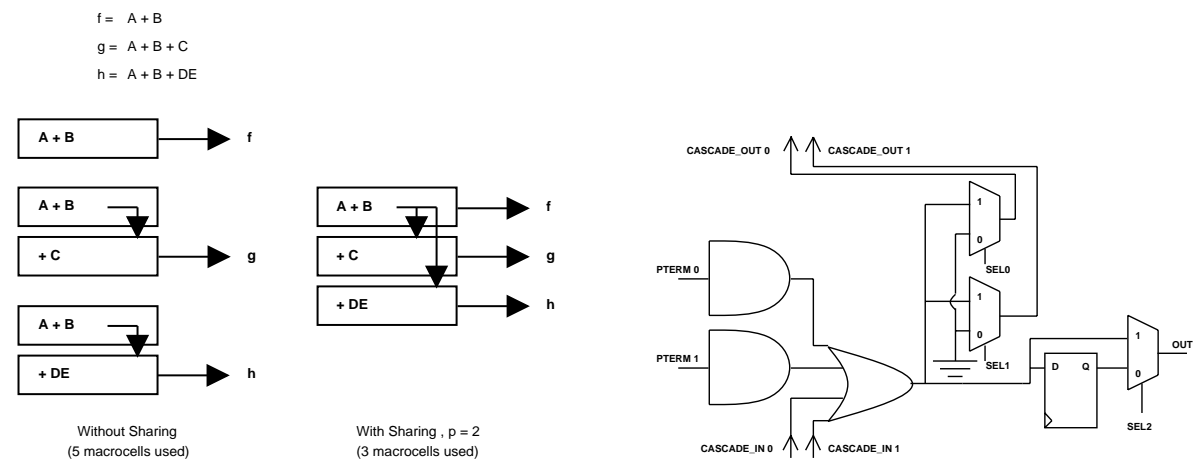

a) Case where multiple expanders is useful b) Basic macrocell with multiple expanders

Fig. 4. Multiple parallel expanders

\section{Mapping to Product Term Memory: pMapster Algorithm}

In order to take advantage of the above architectural enhancements, a new technology mapping tool, pMapster, was implemented.

\subsection{Basic Algorithm}

The objective of our algorithm is to determine which nodes in a circuit are suitable for implementation as product terms, and to remove them from the network. The removed nodes are implemented using product term memory, and the remaining nodes are implemented using 4-LUTs.

The algorithm is outlined in Figure 5. Each internal node in the network is considered as a potential seed node. For each potential node, we repeatedly 


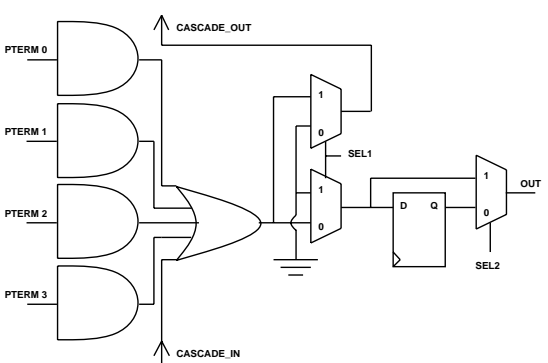

a) Granularity $g=4$

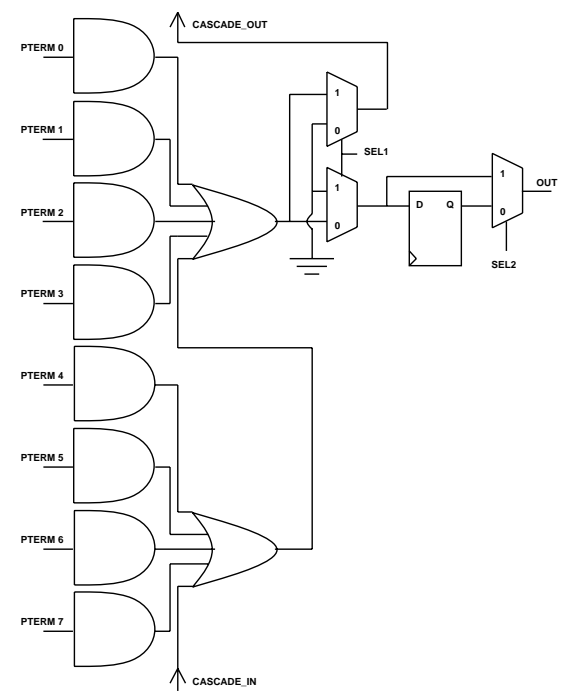

b) Granularity $g=8$

Fig. 2. Macrocell granularity

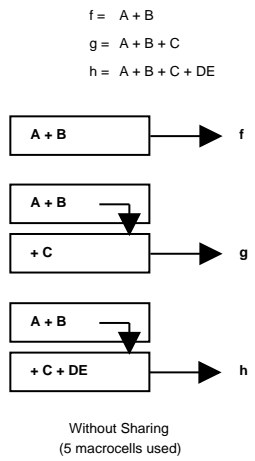

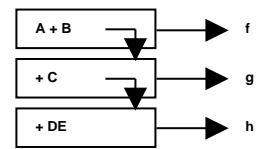

With Sharing

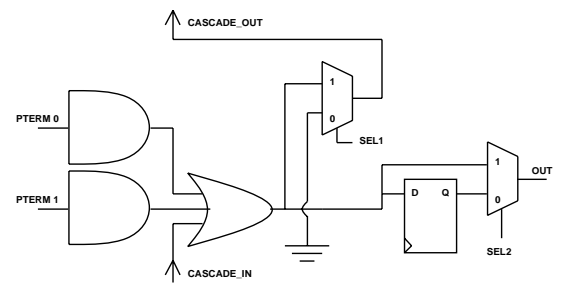

a) Case where output sharing is useful

b) Basic macrocell with output sharing

Fig. 3. Sharing macrocell outputs 
multiple macrocell outputs are OR'ed together. This is done through a structure called the parallel expander.

There is also extra logic inside the macrocell that permits the macrocell to compute the XOR of the two product terms instead of an OR. Finally, the output of the macrocell can be programmably configured to be registered or non-registered. Figure 1 shows a schematic of a simplified APEX20K macrocell.

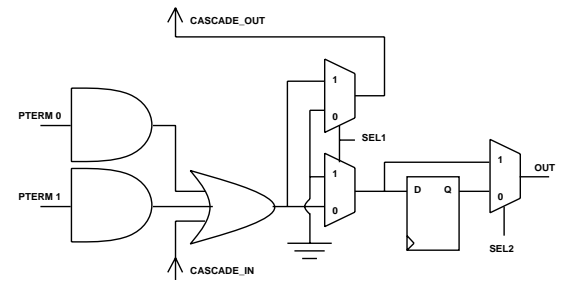

Fig. 1. A simplified APEX20K macrocell

\subsection{Architectural Enhancements}

Granularity of the Macrocell: When implementing functions of a large number of product terms, many macrocells need to be cascaded (using the parallel expander) to implement the desired number of product terms. However, this incurs an incremental delay for every macrocell that is cascaded. A solution is to increase the number of product terms per macrocell (the granularity of the macrocell) so that functions with more product terms can be more efficiently implemented. Unfortunately, increasing the granularity also decreases the efficiency with which smaller functions are implemented. Figure 2 illustrate the granularity enhancements.

Sharing Macrocell Outputs: In the APEX20K, each macrocell can either direct the sum of the product terms to the macrocell's output or to the next macrocell via the parallel expander, but not both simultaneously. This may lead to product terms being used inefficiently.

In cases where the product term block must implement a number of functions which are incrementally similar, having a macrocell capable of sharing its output with the next macrocell may be useful. Macrocells can then be chained together to efficiently implement these functions. Figure 3a) shows how a macrocell with output sharing could be useful. Figure $3 \mathrm{~b}$ ) is a schematic of a basic macrocell with the enhanced output circuitry.

Multiple Parallel Expanders: Since each macrocell has only one connection to the adjacent macrocell, product terms generated in one macrocell can only 
However, this chip area need not be wasted if these unused arrays are used to implement logic. Unused arrays could be configured as large ROM multipleinput, multiple-output lookup tables. Wilton [5] proposes an algorithm, SMAP, which packs logic into unused memory arrays. Cong and Xu's EMB_Pack [6] is another example of an algorithm that packs logic into memory arrays.

A recent innovation in on-chip storage is the addition of a product term mode for FPGA memories. Heile and Leaver [7] describe one such product term based architecture, found in the Altera APEX20K family of FPGAs. Product term memories can either operate as regular RAM (which can be used for storage or implementing logic), or as a collection of macrocells that implement logic in a manner similar to PLAs. In the Altera APEX20K architecture, an array operating in product term mode can implement between 16 outputs of two product terms each, to one output of 32 product terms. The number of outputs is variable as long as the total number of product terms implemented by the macrocells does not exceed 32 [2].

In the product term memory in [7], the OR plane is implemented using a collection of macrocells. Compared to traditional PLA architectures, this results in a far less flexible mechanism for combining product terms. To compensate for this, a sharing mechanism is provided, by which product terms can be shared between macrocells. The structure of this sharing mechanism is critical; if it is too flexible, it will slow the memory unacceptably, while if it is not flexible enough, it will limit the achievable logic density. In this paper, we present several alternative sharing schemes, and quantify their ability to implement logic.

In order to perform this evaluation, we require a technology mapping tool that can pack logic into the memory arrays. Traditional PLA mappers [8,9] are not sufficient, since they don't take into account the specific OR plane (macrocell) architecture. Krishnamoorthy details an algorithm targeting a product term memory [10], however, it does not take into account the product term sharing mechanism. Thus, a second contribution of this paper is a new algorithm, pMapster, which is flexible enough to map logic to product term memories with a variety of sharing mechanisms.

\section{Macrocell Architectures}

In this section we present several alternate parameterized macrocell architectures.

\subsection{Base Architecture}

The Altera APEX20K product term architecture uses the large embedded RAM arrays to implement an AND array similar to that found in a PLA [11]. The column outputs are connected to macrocell structures that implement the OR array function of a PLA. Each macrocell implements a sum of a fixed number of product terms, usually two. In cases where more than two product terms are needed to implement a function, adjacent macrocells can be cascaded such that 


\title{
Macrocell Architectures for Product Term Embedded Memory Arrays
}

\author{
Ernie Lin and Steven J.E. Wilton \\ Department of Electrical and Computer Engineering \\ University of British Columbia, \\ Vancouver, B.C., Canada, \\ \{erniel|stevew $\}$ Oece.ubc.ca \\ http: //ww. ece.ubc.ca/ ${ }^{\sim}$ stevew
}

\begin{abstract}
We examine ways to increase product term usage efficiency and propose several new sharing architectures that addresses this problem. We also present a technology mapping algorithm for product term based FPGA embedded memory arrays. Our algorithm, pMapster, is used to investigate the effects of macrocell granularity and macrocell sharing on the amount of logic that can be packed into a product term embedded memory array.
\end{abstract}

\section{Introduction}

On-chip memory has become an essential component of modern FPGAs. FPGAs are being used to implement entire systems, as opposed to smaller glue-logic type subcircuits that FPGAs have traditionally been used to implement; large systems typically require storage and memories, something that smaller subcircuits usually do not. Though memory could be implemented off-chip, on-chip storage can lead to higher clock frequencies and relaxed I/O pin requirements.

On-chip storage architectures can be classified as either fine-grained or coarsegrained. In fine-grained architectures, such as the Xilinx 4000, each look-up table can be configured as a tiny RAM, and many of these RAMs can be combined to create larger user memories [1]. Coarse-grained architectures use large memory arrays embedded in the FPGA. For example, Altera APEX20KE FPGAs have between 12 and 216 2-Kbit arrays [2], while Xilinx Virtex-E FPGAs have between 16 and 1444 - Kbit arrays [3].

The coarse-grained approach to memory architecture results in significantly denser memory implementations, due to less overhead per bit of memory [4]. However, this approach requires the FPGA manufacturer to partition the chip into memory and logic regions when the FPGA is designed. Since circuits have widely-varying memory demands, this "average case" partitioning may result in poor device utilization for logic-intensive applications. Specifically, if the on-chip memory is not utilized completely or not at all, the chip area devoted to these unused memory arrays is wasted. 